\title{
Numerical Solutions of Eleventh Order Boundary Value Problems Using Piecewise Polynomials
}

\author{
Md. Bellal Hossain ${ }^{1}$, Md. Shafiqul Islam ${ }^{2}$, Md. Azizur Rahman ${ }^{3}$ \\ ${ }^{I}$ (Department of Mathematics, Patuakhali Science and Technology University, Dumki, Patuakhali-8602, \\ Bangladesh) \\ ${ }_{2}^{2}$ (Department of Mathematics, University of Dhaka, Dhaka - 1000, Bangladesh) \\ ${ }^{3}$ (Department of Mathematics and Statistics, Bangladesh University of Business and Technology, Dhaka-1216, \\ Bangladesh)
}

\begin{abstract}
The aim of this paper is to apply Galerkin weighted residual method for solving eleventh-order BVPS. In this method, we exploit Bernstein and Legendre polynomials as basis functions which are modified into to a new set of basis functions to satisfy the corresponding homogeneous form of boundary conditions where the essential types of boundary conditions are mentioned. The method is formulated as a rigorous matrix form. Examples of both linear and nonlinear BVPs are presented to illustrate the reliability and efficiency of the proposed method. It is observed that the present method is a more effective tool and yields better results.
\end{abstract}

Keywords: Galerkin Method, Eleventh-order Linear and Nonlinear BVPs, Bernstein and Legendre Polynomials.

\section{Introduction}

Agarwal [1] discussed the theorems stating the conditions for the existence and uniqueness of solutions of high order boundary value problems (BVP) without numerical methods. In the literature of BVPs we observe that the higher order differential equations arise in some branches of applied mathematics, engineering and many other fields of advanced physical sciences [2-3]. Particularly, eighth, tenth and more even higher order BVP arise in hydromagnetic stability analysis [4]. Thus, some researchers have solved high even order BVP numerically; e.g. Siddiqi and Twizell [5] solved eighth order BVPs with spline functions while Wazwaz [6] considered tenth order problems by the modified decomposition method. But few researchers have paid their attention to solve eleventh order BVP. Such as Siddiqi et al [7] used Variational iteration technique to obtain numerical approximations for eleventh-order BVPs by converting the original problem into a system of integral equations. Very recently Amjad Hussain et al [8] derived the numerical solutions of eleventh-order BVPs using differential transformation method.

In the present paper, the solution of eleventh-order BVP is derived by the Galerkin method [2] with standard (Bernstein and Legendre) polynomials as basis functions. The problem has the following form:

$$
\begin{gathered}
a_{11} \frac{d^{11} u}{d x^{11}} a_{10} \frac{d^{10} u}{d x^{10}}+a_{9} \frac{d^{9} u}{d x^{9}}+a_{8} \frac{d^{8} u}{d x^{8}}+a_{7} \frac{d^{7} u}{d x^{7}}+a_{6} \frac{d^{6} u}{d x^{6}}+a_{5} \frac{d^{5} u}{d x^{5}}+a_{4} \frac{d^{4} u}{d x^{4}}+a_{3} \frac{d^{3} u}{d x^{3}}+a_{2} \frac{d^{2} u}{d x^{2}} \\
+a_{1} \frac{d u}{d x}+a_{0} u=r, a<x<b
\end{gathered}
$$

Subject to the boundary conditions:

$$
\begin{gathered}
u(a)=A_{0}, u(b)=B_{0}, u^{\prime}(a)=A_{1}, u^{\prime}(b)=B_{1}, u^{\prime \prime}(a)=A_{2}, u^{\prime \prime}(b)=B_{2}, u^{\prime \prime \prime}(a)=A_{3}, u^{\prime \prime \prime}(b)=B_{3}, \\
u^{(i v)}(a)=A_{4}, u^{(i v)}(b)=B_{4}, u^{(v)}(a)=A_{5}
\end{gathered}
$$

where $A_{i}, i=0,1,2,3,4,5$ and $B_{j}, j=0,1,2,3,4$ are finite real constants and $a_{i}, i=0,1, \cdots 11$ and $r$ are all continuous functions defined on the interval $[a, b]$. The BVP (1) is solved with the boundary conditions of eqn. (1b).

However, in section 2 of this paper, we give a short description on Bernstein and Legendre polynomials. In section 3, the formulation for solving linear eleventh-order BVP by the Galerkin weighted residual method with Bernstein and Legendre polynomials are presented. Numerical examples and results for both linear and nonlinear BVPs are considered to verify the proposed formulation and the solutions are compared with the existing methods in the literature in section 4. Finally the conclusions of the paper are given in the last section.

\section{Piecewise Polynomials}

\subsection{Bernstein polynomials}

The general form of the Bernstein polynomials of $n$th degree over the interval $[a, b]$ is defined by 


$$
B_{i, n}(x)=\left(\begin{array}{c}
n \\
i
\end{array}\right) \frac{(x-a)^{i}(b-x)^{n-i}}{(b-a)^{n}}, \quad a \leq x \leq b \quad i=0,1,2, \ldots, n .
$$

For example, the first 11 Bernstein polynomials of degree 10 over the interval $[0,1]$ are given bellow:

$$
\begin{array}{lll}
B_{0}(x)=(1-x)^{10} & B_{4}(x)=210(1-x)^{6} x^{4} & B_{8}(x)=45(1-x)^{2} x^{8} \\
B_{1}(x)=10(1-x)^{9} x & B_{5}(x)=252(1-x)^{5} x^{5} & B_{9}(x)=10(1-x) x^{9} \\
B_{2}(x)=45(1-x)^{8} x^{2} & B_{6}(x)=210(1-x)^{4} x^{6} & B_{10}(x)=x^{10} \\
B_{3}(x)=120(1-x)^{7} x^{3} & B_{7}(x)=120(1-x)^{3} x^{7} &
\end{array}
$$

Note that each of these $n+1$ polynomials having degree $n$ satisfies the following properties:

(i) $B_{i, n}(x)=0$ if $i<0$ or $i>n$.

(ii) $\sum_{i=0}^{n} B_{i, n}(x)=1$

(iii) $B_{i, n}(a)=B_{i, n}(b)=0, \quad i=1,2, \ldots, n-1$

For these properties, Bernstein polynomials are used in the trail functions satisfying the corresponding homogeneous form of the essential boundary conditions in the Galerkin method to solve a BVP.

\subsection{Legendre Polynomials}

The general form of the Legendre polynomials of degree $n$ is defined by

$$
P_{n}(x)=\frac{(-1)^{n}}{2^{n}(n !)} \frac{d^{n}}{d x^{n}}\left[\left(1-x^{2}\right)^{n}\right], \quad n \geq 1 .
$$

Now we modify above Legendre polynomials as

$$
p_{n}(x)=\left[\frac{1}{n !} \frac{d^{n}}{d x^{n}}\left(x^{2}-x\right)^{n}-(-1)^{n}\right](x-1), \quad n \geq 1
$$

We write first few modified Legendre polynomials over the interval $[0,1]$ :

$$
\begin{aligned}
p_{1}(x)= & 2 x(x-1), p_{2}(x)=6 x(x-1)^{2}, p_{3}(x)=2 x(x-1)\left(10 x^{2}-15 x+6\right) \\
p_{4}(x)= & 20 x-110 x^{2}+230 x^{3}-210 x^{4}+70 x^{5} \\
p_{5}(x)= & -30 x+240 x^{2}-770 x^{3}+1190 x^{4}-882 x^{5}+252 x^{6} \\
p_{6}(x)= & 42 x-462 x^{2}+2100 x^{3}-4830 x^{4}+5922 x^{5}-3696 x^{6}+924 x^{7} \\
p_{7}(x)= & -56 x+812 x^{2}-4956 x^{3}+15750 x^{4}-28182 x^{5}+28644 x^{6}-15444 x^{7}+3432 x^{8} \\
p_{8}(x)= & 72 x-1332 x^{2}+10500 x^{3}-43890 x^{4}+106722 x^{5}-156156 x^{6}+135564 x^{7}-64350 x^{8} \\
& +12870 x^{9} \\
p_{9}(x)= & -90 x+2070 x^{2}-20460 x^{3}+108570 x^{4}-342342 x^{5}+672672 x^{6}-832260 x^{7} \\
& +630630 x^{8}-267410 x^{9}+48620 x^{10} \\
p_{10}(x)= & 110 x-3080 x^{2}+37290 x^{3}-244530 x^{4}+966966 x^{5}-2438436 x^{6}+4015440 x^{7} \\
- & 4302870 x^{8}+2892890 x^{9}-1108536 x^{10}+184756 x^{11}
\end{aligned}
$$

Since the modified Legendre polynomials have special properties at $x=0$ and $x=1: \quad p_{n}(0)=0$ and $p_{n}(1)=0, n \geq 1$ respectively, so that they can be used as set of basis function to satisfy the corresponding homogeneous form of the Dirichlet boundary conditions to derive the matrix formulation of fourth order BVP over the interval $[0,1]$.

\section{Matrix Formulation of Eleventh-order BVP}

In this section we first derived the matrix formulation for eleventh-order linear BVP and then we extend our idea for solving nonlinear BVP. To solve the boundary value problem (1) by the Galerkin method we approximate $u(x)$ as

$$
\tilde{u}(x)=\theta_{0}(x)+\sum_{i=1}^{n} \alpha_{i} N_{i, n}(x)
$$


Here $\theta_{0}(x)$ is specified by the essential boundary conditions and $N_{i, n}(a)=N_{i, n}(b)=0$ for each $i=1,2, \ldots n$.

Using eqn. (3) into eqn. (1), the weighted residual equations are

$$
\begin{gathered}
\int_{a}^{b}\left[a_{11} \frac{d^{11} u}{d x^{11}} a_{10} \frac{d^{10} u}{d x^{10}}+a_{9} \frac{d^{9} u}{d x^{9}}+a_{8} \frac{d^{8} u}{d x^{8}}+a_{7} \frac{d^{7} u}{d x^{7}}+a_{6} \frac{d^{6} u}{d x^{6}}+a_{5} \frac{d^{5} u}{d x^{5}}+a_{4} \frac{d^{4} u}{d x^{4}}+a_{3} \frac{d^{3} u}{d x^{3}}+a_{2} \frac{d^{2} u}{d x^{2}}\right. \\
\left.+a_{1} \frac{d u}{d x}+a_{0} u-r\right] N_{j, n}(x) d x=0
\end{gathered}
$$

Integrating by parts the terms up to second derivative on the left hand side of (4), we get

$$
\begin{aligned}
& \int_{a}^{b} a_{11} \frac{d^{11} \tilde{u}}{d x^{11}} N_{j, n}(x) d x=-\left[\frac{d}{d x}\left[a_{11} N_{j, n}(x)\right] \frac{d^{9} \tilde{u}}{d x^{9}}\right]_{a}^{b}+\left[\frac{d^{2}}{d x^{2}}\left[a_{11} N_{j, n}(x)\right] \frac{d^{8} \tilde{u}}{d x^{8}}\right]_{a}^{b}-\left[\frac{d^{3}}{d x^{3}}\left[a_{11} N_{j, n}(x)\right] \frac{d^{7} \tilde{u}}{d x^{7}}\right]_{a}^{b} \\
& +\left[\frac{d^{4}}{d x^{4}}\left[a_{11} N_{j, n}(x)\right] \frac{d^{6} \tilde{u}}{d x^{6}}\right]_{a}^{b}-\left[\frac{d^{5}}{d x^{5}}\left[a_{11^{1}} N_{j, n}(x)\right] \frac{d^{5} \tilde{u}}{d x^{5}}\right]_{a}^{b}+\left[\frac{d^{6}}{d x^{6}}\left[c_{11} N_{j, n}(x)\right] \frac{d^{4} \tilde{u}}{d x^{4}}\right]_{a}^{b} \\
& -\left[\frac{d^{7}}{d x^{7}}\left[a_{11} N_{j, n}(x)\right] \frac{d^{3} \tilde{u}}{d x^{3}}\right]_{a}^{b}+\left[\frac{d^{8}}{d x^{8}}\left[a_{11} N_{j, n}(x)\right] \frac{d^{2} \tilde{u}}{d x^{2}}\right]_{a}^{b}-\left[\frac{d^{9}}{d x^{9}}\left[a_{11} N_{j, n}(x)\right] \frac{d \tilde{u}}{d x}\right]_{a}^{b} \\
& +\int_{a}^{b} \frac{d^{10}}{d x^{10}}\left[a_{11} N_{j, n}(x)\right] \frac{d \tilde{u}}{d x} d x
\end{aligned}
$$

$$
\int_{a}^{b} a_{10} \frac{d^{10} \tilde{u}}{d x^{10}} N_{j, n}(x) d x=-\left[\frac{d}{d x}\left[a_{10} N_{j, n}(x)\right] \frac{d^{8} \tilde{u}}{d x^{8}}\right]_{a}^{b}+\left[\frac{d^{2}}{d x^{2}}\left[a_{10} N_{j, n}(x)\right] \frac{d^{7} \tilde{u}}{d x^{7}}\right]_{a}^{b}-\left[\frac{d^{3}}{d x^{3}}\left[a_{10} N_{j, n}(x)\right] \frac{d^{6} \tilde{u}}{d x^{6}}\right]_{a}^{b}
$$

$$
\begin{gathered}
+\left[\frac{d^{4}}{d x^{4}}\left[a_{10} N_{j, n}(x)\right] \frac{d^{5} \tilde{u}}{d x^{5}}\right]_{a}^{b}-\left[\frac{d^{5}}{d x^{5}}\left[a_{10} N_{j, n}(x)\right] \frac{d^{4} \tilde{u}}{d x^{4}}\right]_{a}^{b}+\left[\frac{d^{6}}{d x^{6}}\left[a_{10} N_{j, n}(x)\right] \frac{d^{3} \tilde{u}}{d x^{3}}\right]_{a}^{b} \\
-\left[\frac{d^{7}}{d x^{7}}\left[a_{10} N_{j, n}(x)\right] \frac{d^{2} \tilde{u}}{d x^{2}}\right]_{a}^{b}+\left[\frac{d^{8}}{d x^{8}}\left[a_{10} N_{j, n}(x)\right] \frac{d \tilde{u}}{d x}\right]_{a}^{b}-\int_{a}^{b} \frac{d^{9}}{d x^{9}}\left[a_{10} N_{j, n}(x)\right] \frac{d \tilde{u}}{d x} d x
\end{gathered}
$$

$\int_{a}^{b} a_{9} \frac{d^{9} \tilde{u}}{d x^{9}} N_{j, n}(x) d x=-\left[\frac{d}{d x}\left[a_{9} N_{j, n}(x)\right] \frac{d^{7} \tilde{u}}{d x^{7}}\right]_{a}^{b}+\left[\frac{d^{2}}{d x^{2}}\left[a_{9} N_{j, n}(x)\right] \frac{d^{6} \tilde{u}}{d x^{6}}\right]_{a}^{b}-\left[\frac{d^{3}}{d x^{3}}\left[a_{9} N_{j, n}(x)\right]^{\frac{d^{5} \tilde{u}}{d x^{5}}}\right]_{a}^{b}$

$$
+\left[\frac{d^{4}}{d x^{4}}\left[a_{9} N_{j, n}(x)\right] \frac{d^{4} \tilde{u}}{d x^{4}}\right]_{a}^{b}-\left[\frac{d^{5}}{d x^{5}}\left[a_{9} N_{j, n}(x)\right] \frac{d^{3} \tilde{u}}{d x^{3}}\right]_{a}^{b}+\left[\frac{d^{6}}{d x^{6}}\left[a_{9} N_{j, n}(x)\right] \frac{d^{2} \tilde{u}}{d x^{2}}\right]_{a}^{b}
$$$$
-\left[\frac{d^{7}}{d x^{7}}\left[a_{9} N_{j, n}(x)\right] \frac{d \tilde{u}}{d x}\right]_{a}^{b}+\int_{a}^{b} \frac{d^{8}}{d x^{8}}\left[a_{9} N_{j, n}(x)\right] \frac{d \tilde{u}}{d x} d x
$$

$\int_{a}^{b} a_{8} \frac{d^{8} \tilde{u}}{d x^{8}} N_{j, n}(x) d x=-\left[\frac{d}{d x}\left[a_{8} N_{j, n}(x)\right] \frac{d^{6} \tilde{u}}{d x^{6}}\right]_{a}^{b}+\left[\frac{d^{2}}{d x^{2}}\left[a_{8} N_{j, n}(x)\right] \frac{d^{5} \tilde{u}}{d x^{5}}\right]_{a}^{b}-\left[\frac{d^{3}}{d x^{3}}\left[a_{8} N_{j, n}(x)\right] \frac{d^{4} \tilde{u}}{d x^{4}}\right]_{a}^{b}$ $+\left[\frac{d^{4}}{d x^{4}}\left[a_{8} N_{j, n}(x)\right] \frac{d^{3} \tilde{u}}{d x^{3}}\right]_{a}^{b}-\left[\frac{d^{5}}{d x^{5}}\left[a_{8} N_{j, n}(x)\right] \frac{d^{2} \tilde{u}}{d x^{2}}\right]_{a}^{b}+\left[\frac{d^{6}}{d x^{6}}\left[a_{8} N_{j, n}(x)\right] \frac{d \tilde{u}}{d x}\right]_{a}^{b}$

$$
-\int_{a}^{b} \frac{d^{7}}{d x^{7}}\left[a_{8} N_{j, n}(x)\right] \frac{d \tilde{u}}{d x} d x
$$

$$
\begin{aligned}
\int_{a}^{b} a_{7} \frac{d^{7} \tilde{u}}{d x^{7}} N_{j, n}(x) d x=- & {\left[\frac{d}{d x}\left[a_{7} N_{j, n}(x)\right] \frac{d^{5} \tilde{u}}{d x^{5}}\right]_{a}^{b}+\left[\frac{d^{2}}{d x^{2}}\left[a_{7} N_{j, n}(x)\right] \frac{d^{4} \tilde{u}}{d x^{4}}\right]_{a}^{b}-\left[\frac{d^{3}}{d x^{3}}\left[a_{7} N_{j, n}(x)\right] \frac{d^{3} \tilde{u}}{d x^{3}}\right]_{a}^{b} } \\
+ & {\left[\frac{d^{4}}{d x^{4}}\left[a_{7} N_{j, n}(x)\right] \frac{d^{2} \tilde{u}}{d x^{2}}\right]_{a}^{b}-\left[\frac{d^{5}}{d x^{5}}\left[a_{7} N_{j, n}(x)\right] \frac{d \tilde{u}}{d x}\right]_{a}^{b}+\int_{a}^{b} \frac{d^{6}}{d x^{6}}\left[a_{7} N_{j, n}(x)\right] \frac{d \tilde{u}}{d x} d x(9) }
\end{aligned}
$$




$$
\begin{aligned}
& \int_{a}^{b} a_{6} \frac{d^{6} \tilde{u}}{d x^{6}} N_{j, n}(x) d x=-\left[\frac{d}{d x}\left[a_{6} N_{j, n}(x)\right] \frac{d^{4} \tilde{u}}{d x^{4}}\right]_{a}^{b}+\left[\frac{d^{2}}{d x^{2}}\left[a_{6} N_{j, n}(x)\right] \frac{d^{3} \tilde{u}}{d x^{3}}\right]_{a}^{b}-\left[\frac{d^{3}}{d x^{3}}\left[a_{6} N_{j, n}(x)\right] \frac{d^{2} \tilde{u}}{d x^{2}}\right]_{a}^{b} \\
& +\left[\frac{d^{4}}{d x^{4}}\left[a_{6} N_{j, n}(x)\right] \frac{d \tilde{u}}{d x}\right]_{a}^{b}-\int_{a}^{b} \frac{d^{5}}{d x^{5}}\left[a_{6} N_{j, n}(x)\right] \frac{d \tilde{u}}{d x} d x \\
& \int_{a}^{b} a_{5} \frac{d^{5} \tilde{u}}{d x^{5}} N_{j, n}(x) d x=-\left[\frac{d}{d x}\left[a_{5} N_{j, n}(x)\right] \frac{d^{3} \tilde{u}}{d x^{3}}\right]_{a}^{b}+\left[\frac{d^{2}}{d x^{2}}\left[a_{5} N_{j, n}(x)\right] \frac{d^{2} \tilde{u}}{d x^{2}}\right]_{a}^{b}-\left[\frac{d^{3}}{d x^{3}}\left[a_{5} N_{j, n}(x)\right] \frac{d \tilde{u}}{d x}\right]_{a}^{b} \\
& +\int_{a}^{b} \frac{d^{4}}{d x^{4}}\left[a_{5} N_{j, n}(x)\right] \frac{d \tilde{u}}{d x} d x \\
& \int_{a}^{b} a_{4} \frac{d^{4} \tilde{u}}{d x^{4}} N_{j, n}(x) d x=-\left[\frac{d}{d x}\left[a_{4} N_{j, n}(x)\right] \frac{d^{2} \tilde{u}}{d x^{2}}\right]_{a}^{b}+\left[\frac{d^{2}}{d x^{2}}\left[a_{4} N_{j, n}(x)\right] \frac{d \tilde{u}}{d x}\right]_{a}^{b}-\int_{a}^{b} \frac{d^{3}}{d x^{3}}\left[a_{4} N_{j, n}(x)\right] \frac{d \tilde{u}}{d x} d x \\
& \int_{a}^{b} a_{3} \frac{d^{3} \tilde{u}}{d x^{3}} N_{j, n}(x) d x==-\left[\frac{d}{d x}\left[a_{3} N_{j, n}(x)\right] \frac{d \tilde{u}}{d x}\right]_{a}^{b}+\int_{a}^{b} \frac{d^{2}}{d x^{2}}\left[a_{3} N_{j, n}(x)\right] \frac{d \tilde{u}}{d x} d x \\
& \int_{a}^{b} a_{2} \frac{d^{2} \tilde{u}}{d x^{2}} N_{j, n}(x) d x=-\int_{a}^{b} \frac{d}{d x}\left[a_{2} N_{j, n}(x)\right] \frac{d \tilde{u}}{d x} d x
\end{aligned}
$$

Putting eqns. (5) - (14) into eqn. (4) and using approximation for $\tilde{u}(x)$ given in eqn. (3) and after applying the boundary conditions given in eqn. (1b) and rearranging the terms for the resulting equations we get a system of equations in matrix form as

$$
\sum_{i=1}^{n} D_{i, j} \alpha_{i}=F_{j}, j=1,2, \ldots, n
$$

where

$$
\begin{aligned}
D_{i, j}=\int_{a}^{b}\{ & \left\{\frac{d^{10}}{d x^{10}}\left[a_{11} N_{j, n}(x)\right]-\frac{d^{9}}{d x^{9}}\left[a_{10} N_{j, n}(x)\right]+\frac{d^{8}}{d x^{8}}\left[a_{9} N_{j, n}(x)\right]-\frac{d^{7}}{d x^{7}}\left[a_{8} N_{j, n}(x)\right]+\frac{d^{6}}{d x^{6}}\left[a_{7} N_{j, n}(x)\right]\right. \\
& +\frac{d^{2}}{d x^{2}}\left[a_{3} N_{j, n}(x)\right]-\frac{d}{d x}\left[a_{2} N_{j, n}(x)\right]-\frac{d^{5}}{d x^{5}}\left[a_{6} N_{j, n}(x)\right]+\frac{d^{4}}{d x^{4}}\left[a_{5} N_{j, n}(x)\right]-\frac{d^{3}}{d x^{3}}\left[a_{4} N_{j, n}(x)\right] \\
& \left.\left.+a_{1} N_{j, n}(x)\right] \frac{d}{d x}\left[N_{i, n}(x)\right]+a_{0} N_{i, n}(x) N_{j, n}(x)\right\} d x-\left[\frac{d}{d x}\left[a_{11^{1}} N_{j, n}(x)\right] \frac{d^{9}}{d x^{9}}\left[N_{i, n}(x)\right]\right]_{x=b} \\
& +\left[\frac{d}{d x}\left[a_{11} N_{j, n}(x)\right] \frac{d^{9}}{d x^{9}}\left[N_{i, n}(x)\right]\right]_{x=a}+\left[\frac{d^{2}}{d x^{2}}\left[a_{11} N_{j, n}(x)\right] \frac{d^{8}}{d x^{8}}\left[N_{i, n}(x)\right]\right]_{x=b} \\
& -\left[\frac{d^{2}}{d x^{2}}\left[a_{11} N_{j, n}(x)\right] \frac{d^{8}}{d x^{8}}\left[N_{i, n}(x)\right]\right]_{x=a}-\left[\frac{d^{3}}{d x^{3}}\left[a_{11} N_{j, n}(x)\right] \frac{d^{7}}{d x^{7}}\left[N_{i, n}(x)\right]\right]_{x=b} \\
& +\left[\frac{d^{3}}{d x^{3}}\left[a_{11} N_{j, n}(x)\right] \frac{d^{7}}{d x^{7}}\left[N_{i, n}(x)\right]\right]_{x=a}+\left[\frac{d^{4}}{d x^{4}}\left[a_{11} N_{j, n}(x)\right] \frac{d^{6}}{d x^{6}}\left[N_{i, n}(x)\right]\right]_{x=b} \\
& -\left[\frac{d^{4}}{d x^{4}}\left[a_{11} N_{j, n}(x)\right] \frac{d^{6}}{d x^{6}}\left[N_{i, n}(x)\right]\right]_{x=a}-\left[\frac{d^{5}}{d x^{5}}\left[a_{11} N_{j, n}(x)\right] \frac{d^{5}}{d x^{5}}\left[N_{i, n}(x)\right]\right]_{x=b} \\
& -\left[\frac{d}{d x}\left[a_{10} N_{j, n}(x)\right] \frac{d^{8}}{d x^{8}}\left[N_{i, n}(x)\right]\right]_{x=b}+\left[\frac{d}{d x}\left[a_{10} N_{j, n}(x)\right] \frac{d^{8}}{d x^{8}}\left[N_{i, n}(x)\right]\right]_{x=a} \\
+ & {\left[\frac{d^{2}}{d x^{2}}\left[a_{10} N_{j, n}(x)\right] \frac{d^{7}}{d x^{7}}\left[N_{i, n}(x)\right]\right]_{x=b}-\left[\frac{d^{2}}{d x^{2}}\left[a_{10} N_{j, n}(x)\right] \frac{d^{7}}{d x^{7}}\left[N_{i, n}(x)\right]\right]_{x=a} }
\end{aligned}
$$




$$
\begin{aligned}
& -\left[\frac{d^{3}}{d x^{3}}\left[a_{10} N_{j, n}(x)\right] \frac{d^{6}}{d x^{6}}\left[N_{i, n}(x)\right]\right]_{x=b}+\left[\frac{d^{3}}{d x^{3}}\left[a_{10} N_{j, n}(x)\right] \frac{d^{6}}{d x^{6}}\left[N_{i, n}(x)\right]\right]_{x=a} \\
& +\left[\frac{d^{4}}{d x^{4}}\left[a_{10} N_{j, n}(x)\right] \frac{d^{5}}{d x^{5}}\left[N_{i, n}(x)\right]\right]_{x=b}-\left[\frac{d}{d x}\left[a_{9} N_{j, n}(x)\right] \frac{d^{7}}{d x^{7}}\left[N_{i, n}(x)\right]\right]_{x=b} \\
& +\left[\frac{d}{d x}\left[a_{9} N_{j, n}(x)\right] \frac{d^{7}}{d x^{7}}\left[N_{i, n}(x)\right]\right]_{x=b}+\left[\frac{d^{2}}{d x^{2}}\left[a_{9} N_{j, n}(x)\right] \frac{d^{6}}{d x^{6}}\left[N_{i, n}(x)\right]\right]_{x=b} \\
& -\left[\frac{d^{2}}{d x^{2}}\left[a_{9} N_{j, n}(x)\right] \frac{d^{6}}{d x^{6}}\left[N_{i, n}(x)\right]\right]_{x=a}-\left[\frac{d^{3}}{d x^{3}}\left[a_{9} N_{j, n}(x)\right] \frac{d^{5}}{d x^{5}}\left[N_{i, n}(x)\right]\right]_{x=b} \\
& -\left[\frac{d}{d x}\left[a_{8} N_{j, n}(x)\right] \frac{d^{6}}{d x^{6}}\left[N_{i, n}(x)\right]\right]_{x=b}+\left[\frac{d}{d x}\left[a_{8} N_{j, n}(x)\right] \frac{d^{6}}{d x^{6}}\left[N_{i, n}(x)\right]\right]_{x=a} \\
& +\left[\frac{d^{2}}{d x^{2}}\left[a_{8} N_{j, n}(x)\right] \frac{d^{5}}{d x^{5}}\left[N_{i, n}(x)\right]\right]_{x=b}-\left[\frac{d}{d x}\left[a_{7} N_{j, n}(x)\right] \frac{d^{5}}{d x^{5}}\left[N_{i, n}(x)\right]\right]_{x=b} \\
& F_{j}=\int_{a}^{b}\left\{s N_{j, n}(x)+\left[-\frac{d^{10}}{d x^{10}}\left[a_{11} N_{j, n}(x)\right]+\frac{d^{9}}{d x^{9}}\left[a_{10} N_{j, n}(x)\right]-\frac{d^{8}}{d x^{8}}\left[a_{9} N_{j, n}(x)\right]+\frac{d^{7}}{d x^{7}}\left[a_{8} N_{j, n}(x)\right]\right.\right. \\
& -\frac{d^{6}}{d x^{6}}\left[a_{7} N_{j, n}(x)\right]+\frac{d^{5}}{d x^{5}}\left[a_{6} N_{j, n}(x)\right]-\frac{d^{4}}{d x^{4}}\left[a_{5} N_{j, n}(x)\right]+\frac{d^{3}}{d x^{3}}\left[a_{4} N_{j, n}(x)\right]+\frac{d^{2}}{d x^{2}}\left[a_{3} N_{j, n}(x)\right] \\
& \left.\left.+\frac{d}{d x}\left[a_{2} N_{j, n}(x)\right]-a_{1} N_{j, n}(x)\right] \frac{d \theta_{0}}{d x}-a_{0} \theta_{0} N_{j, n}(x)\right\} d x+\left[\frac{d}{d x}\left[a_{11} N_{j, n}(x)\right] \frac{d^{9} \theta_{0}}{d x^{9}}\right]_{x=b} \\
& -\left[\frac{d}{d x}\left[a_{11} N_{j, n}(x)\right] \frac{d^{9} \theta_{0}}{d x^{9}}\right]_{x=0}-\left[\frac{d^{2}}{d x^{2}}\left[a_{11} N_{j, n}(x)\right] \frac{d^{8} \theta_{0}}{d x^{8}}\right]_{x=b}+\left[\frac{d^{2}}{d x^{2}}\left[a_{11} N_{j, n}(x)\right] \frac{d^{8} \theta_{0}}{d x^{8}}\right]_{x=a} \\
& +\left[\frac{d^{3}}{d x^{3}}\left[a_{11} N_{j, n}(x)\right] \frac{d^{7} \theta_{0}}{d x^{7}}\right]_{x=b}-\left[\frac{d^{3}}{d x^{3}}\left[a_{11} N_{j, n}(x)\right] \frac{d^{7} \theta_{0}}{d x^{7}}\right]_{x=a}-\left[\frac{d^{4}}{d x^{4}}\left[a_{11} N_{j, n}(x)\right] \frac{d^{6} \theta_{0}}{d x^{6}}\right]_{x=b} \\
& +\left[\frac{d^{4}}{d x^{4}}\left[a_{11} N_{j, n}(x)\right] \frac{d^{6} \theta_{0}}{d x^{6}}\right]_{x=a}+\left[\frac{d^{5}}{d x^{5}}\left[a_{11} N_{j, n}(x)\right] \frac{d^{5} \theta_{0}}{d x^{5}}\right]_{x=b}+\left[\frac{d}{d x}\left[a_{10} N_{j, n}(x)\right] \frac{d^{8} \theta_{0}}{d x^{8}}\right]_{x=b} \\
& -\left[\frac{d}{d x}\left[a_{10} N_{j, n}(x)\right] \frac{d^{8} \theta_{0}}{d x^{8}}\right]_{x=a}-\left[\frac{d^{2}}{d x^{2}}\left[a_{10} N_{j, n}(x)\right] \frac{d^{7} \theta_{0}}{d x^{7}}\right]_{x=b}+\left[\frac{d^{2}}{d x^{2}}\left[a_{10} N_{j, n}(x)\right] \frac{d^{7} \theta_{0}}{d x^{7}}\right]_{x=a} \\
& +\left[\frac{d^{3}}{d x^{3}}\left[a_{10} N_{j, n}(x)\right] \frac{d^{6} \theta_{0}}{d x^{6}}\right]_{x=b}-\left[\frac{d^{3}}{d x^{3}}\left[a_{10} N_{j, n}(x)\right] \frac{d^{6} \theta_{0}}{d x^{6}}\right]_{x=a}-\left[\frac{d^{4}}{d x^{4}}\left[a_{10} N_{j, n}(x)\right] \frac{d^{5} \theta_{0}}{d x^{5}}\right]_{x=b} \\
& +\left[\frac{d}{d x}\left[a_{9} N_{j, n}(x)\right] \frac{d^{7} \theta_{0}}{d x^{7}}\right]_{x=b}-\left[\frac{d}{d x}\left[a_{9} N_{j, n}(x)\right] \frac{d^{7} \theta_{0}}{d x^{7}}\right]_{x=a}-\left[\frac{d^{2}}{d x^{2}}\left[a_{9} N_{j, n}(x)\right] \frac{d^{6} \theta_{0}}{d x^{6}}\right]_{x=b} \\
& +\left[\frac{d^{2}}{d x^{2}}\left[a_{9} N_{j, n}(x)\right] \frac{d^{6} \theta_{0}}{d x^{6}}\right]_{x=a}+\left[\frac{d^{3}}{d x^{3}}\left[a_{9} N_{j, n}(x)\right] \frac{d^{5} \theta_{0}}{d x^{5}}\right]_{x=b}+\left[\frac{d}{d x}\left[a_{8} N_{j, n}(x)\right] \frac{d^{6} \theta_{0}}{d x^{6}}\right]_{x=b} \\
& -\left[\frac{d}{d x}\left[a_{8} N_{j, n}(x)\right] \frac{d^{6} \theta_{0}}{d x^{6}}\right]_{x=a}-\left[\frac{d^{2}}{d x^{2}}\left[a_{8} N_{j, n}(x)\right] \frac{d^{5} \theta_{0}}{d x^{5}}\right]_{x=b}+\left[\frac{d}{d x}\left[a_{7} N_{j, n}(x)\right] \frac{d^{5} \theta_{0}}{d x^{5}}\right]_{x=b} \\
& -\left[\frac{d^{5}}{d x^{5}}\left[a_{11} N_{j, n}(x)\right]\right]_{x=a} \times A_{5}-\left[\frac{d^{6}}{d x^{6}}\left[a_{11} N_{j, n}(x)\right]\right]_{x=b} \times B_{4}+\left[\frac{d^{6}}{d x^{6}}\left[a_{11} N_{j, n}(x)\right]\right]_{x=a} \times A_{4} \\
& +\left[\frac{d^{7}}{d x^{7}}\left[a_{11} N_{j, n}(x)\right]\right]_{x=b} \times B_{3}-\left[\frac{d^{7}}{d x^{7}}\left[a_{11} N_{j, n}(x)\right]\right]_{x=a} \times A_{3}-\left[\frac{d^{8}}{d x^{8}}\left[a_{11} N_{j, n}(x)\right]\right]_{x=b} \times B_{2}
\end{aligned}
$$




$$
\begin{aligned}
& +\left[\frac{d^{8}}{d x^{8}}\left[a_{11} N_{j, n}(x)\right]\right]_{x=a} \times A_{2}+\left[\frac{d^{9}}{d x^{9}}\left[a_{11} N_{j, n}(x)\right]\right]_{x=b} \times B_{1}-\left[\frac{d^{9}}{d x^{9}}\left[a_{11} N_{j, n}(x)\right]\right]_{x=a} \times A_{1} \\
& +\left[\frac{d^{4}}{d x^{4}}\left[a_{10} N_{j, n}(x)\right]\right]_{x=a} \times A_{5}+\left[\frac{d^{5}}{d x^{5}}\left[a_{10} N_{j, n}(x)\right]\right]_{x=b} \times B_{4}-\left[\frac{d^{5}}{d x^{5}}\left[a_{10} N_{j, n}(x)\right]\right]_{x=a} \times A_{4} \\
& -\left[\frac{d^{6}}{d x^{6}}\left[a_{10} N_{j, n}(x)\right]\right]_{x=b} \times B_{3^{+}}\left[\frac{d^{6}}{d x^{6}}\left[a_{10} N_{j, n}(x)\right]\right]_{x=a} \times A_{3^{+}}\left[\frac{d^{7}}{d x^{7}}\left[a_{10} N_{j, n}(x)\right]\right]_{x=b} \times B_{2} \\
& -\left[\frac{d^{7}}{d x^{7}}\left[a_{10} N_{j, n}(x)\right]\right]_{x=a} \times A_{2}-\left[\frac{d^{8}}{d x^{8}}\left[a_{10} N_{j, n}(x)\right]\right]_{x=b} \times B_{1}+\left[\frac{d^{8}}{d x^{8}}\left[a_{10} N_{j, n}(x)\right]\right]_{x=a} \times A_{1} \\
& -\left[\frac{d^{3}}{d x^{3}}\left[a_{9} N_{j, n}(x)\right]\right]_{x=a} \times A_{5}-\left[\frac{d^{4}}{d x^{4}}\left[a_{9} N_{j, n}(x)\right]\right]_{x=b} \times B_{4}+\left[\frac{d^{4}}{d x^{4}}\left[a_{9} N_{j, n}(x)\right]\right]_{x=a} \times A_{4} \\
& +\left[\frac{d^{5}}{d x^{5}}\left[a_{9} N_{j, n}(x)\right]\right]_{x=b} \times B_{3}-\left[\frac{d^{5}}{d x^{5}}\left[a_{9} N_{j, n}(x)\right]\right]_{x=a} \times A_{3}-\left[\frac{d^{6}}{d x^{6}}\left[a_{9} N_{j, n}(x)\right]\right]_{x=b} \times B_{2} \\
& +\left[\frac{d^{6}}{d x^{6}}\left[a_{9} N_{j, n}(x)\right]\right]_{x=a} \times A_{2}+\left[\frac{d^{7}}{d x^{7}}\left[a_{9} N_{j, n}(x)\right]\right]_{x=b} \times B_{1}-\left[\frac{d^{7}}{d x^{7}}\left[a_{9} N_{j, n}(x)\right]\right]_{x=a} \times A_{1} \\
& -\left[\frac{d^{2}}{d x^{2}}\left[a_{8} N_{j, n}(x)\right]\right]_{x=a} \times A_{5}+\left[\frac{d^{3}}{d x^{3}}\left[a_{8} N_{j, n}(x)\right]\right]_{x=b} \times B_{4}-\left[\frac{d^{3}}{d x^{3}}\left[a_{8} N_{j, n}(x)\right]\right]_{x=a} \times A_{4} \\
& -\left[\frac{d^{4}}{d x^{4}}\left[a_{8} N_{j, n}(x)\right]\right]_{x=b} \times B_{3}+\left[\frac{d^{4}}{d x^{4}}\left[a_{8} N_{j, n}(x)\right]\right]_{x=a} \times A_{3}+\left[\frac{d^{5}}{d x^{5}}\left[a_{8} N_{j, n}(x)\right]\right]_{x=b} \times B_{2} \\
& -\left[\frac{d^{5}}{d x^{5}}\left[a_{8} N_{j, n}(x)\right]\right]_{x=a} \times A_{2}-\left[\frac{d^{6}}{d x^{6}}\left[a_{8} N_{j, n}(x)\right]\right]_{x=b} \times B_{1}+\left[\frac{d^{6}}{d x^{6}}\left[a_{8} N_{j, n}(x)\right]\right]_{x=a} \times A_{1} \\
& -\left[\frac{d}{d x}\left[a_{7} N_{j, n}(x)\right]\right]_{x=a} \times A_{5}-\left[\frac{d^{2}}{d x^{2}}\left[a_{7} N_{j, n}(x)\right]\right]_{x=b} \times B_{4}+\left[\frac{d^{2}}{d x^{2}}\left[a_{7} N_{j, n}(x)\right]\right]_{x=a} \times A_{4} \\
& +\left[\frac{d^{3}}{d x^{3}}\left[a_{7} N_{j, n}(x)\right]\right]_{x=b} \times B_{3}-\left[\frac{d^{3}}{d x^{3}}\left[a_{7} N_{j, n}(x)\right]\right]_{x=a} \times A_{3}-\left[\frac{d^{4}}{d x^{4}}\left[a_{7} N_{j, n}(x)\right]\right]_{x=b} \times B_{2} \\
& +\left[\frac{d^{4}}{d x^{4}}\left[a_{7} N_{j, n}(x)\right]\right]_{x=a} \times A_{2}+\left[\frac{d^{5}}{d x^{5}}\left[a_{7} N_{j, n}(x)\right]\right]_{x=b} \times B_{1}-\left[\frac{d^{5}}{d x^{5}}\left[a_{7} N_{j, n}(x)\right]\right]_{x=a} \times A_{1} \\
& +\left[\frac{d}{d x}\left[a_{6} N_{j, n}(x)\right]\right]_{x=b} \times B_{4}-\left[\frac{d}{d x}\left[a_{6} N_{j, n}(x)\right]\right]_{x=a} \times A_{4}-\left[\frac{d^{2}}{d x^{2}}\left[a_{6} N_{j, n}(x)\right]\right]_{x=b} \times B_{3} \\
& +\left[\frac{d^{2}}{d x^{2}}\left[a_{6} N_{j, n}(x)\right]\right]_{x=a} \times A_{3}+\left[\frac{d^{3}}{d x^{3}}\left[a_{6} N_{j, n}(x)\right]\right]_{x=b} \times B_{2}-\left[\frac{d^{3}}{d x^{3}}\left[a_{6} N_{j, n}(x)\right]\right]_{x=a} \times A_{2} \\
& -\left[\frac{d^{4}}{d x^{4}}\left[a_{6} N_{j, n}(x)\right]\right]_{x=b} \times B_{1}+\left[\frac{d^{4}}{d x^{4}}\left[a_{6} N_{j, n}(x)\right]\right]_{x=a} \times A_{1}+\left[\frac{d}{d x}\left[a_{5} N_{j, n}(x)\right]\right]_{x=b} \times B_{3} \\
& -\left[\frac{d}{d x}\left[a_{5} N_{j, n}(x)\right]\right]_{x=a} \times A_{3}-\left[\frac{d^{2}}{d x^{2}}\left[a_{5} N_{j, n}(x)\right]\right]_{x=b} \times B_{2}+\left[\frac{d^{2}}{d x^{2}}\left[a_{5} N_{j, n}(x)\right]\right]_{x=a} \times A_{2} \\
& +\left[\frac{d^{3}}{d x^{3}}\left[a_{5} N_{j, n}(x)\right]\right]_{x=b} \times B_{1}-\left[\frac{d^{3}}{d x^{3}}\left[a_{5} N_{j, n}(x)\right]\right]_{x=a} \times A_{1}+\left[\frac{d}{d x}\left[a_{4} N_{j, n}(x)\right]\right]_{x=b} \times B_{2} \\
& -\left[\frac{d}{d x}\left[a_{4} N_{j, n}(x)\right]\right]_{x=a} \times A_{2}-\left[\frac{d^{2}}{d x^{2}}\left[a_{4} N_{j, n}(x)\right]\right]_{x=b} \times B_{1}+\left[\frac{d^{2}}{d x^{2}}\left[a_{4} N_{j, n}(x)\right]\right]_{x=a} \times A_{1}
\end{aligned}
$$




$$
+\left[\frac{d}{d x}\left[a_{3} N_{j, n}(x)\right]\right]_{x=b} \times B_{1}-\left[\frac{d}{d x}\left[a_{3} N_{j, n}(x)\right]\right]_{x=a} \times A_{1}
$$

Solving the system (15a), we find the values of the parameters $\alpha_{i}$ and then substituting these parameters into eqn. (3), we get the approximate solution of the BVP (1). For nonlinear eleventh-order BVP, we first compute the initial values on neglecting the nonlinear terms and using the system (15). Then using the Newton's iterative method we find the numerical approximations for desired nonlinear BVP. This formulation is described through the numerical examples in the next section.

\section{Numerical Examples And Results}

To test the applicability of the proposed method, we consider two linear and one nonlinear problem which are available in the existing literature. For all examples, the solutions obtained by the proposed method are compared with the exact solutions. All the calculations are performed by MATLAB 10. The convergence of linear BVP is calculated by

$$
E=\left|\tilde{u}_{n+1}(x)-\tilde{u}_{n}(x)\right|<10^{-13}
$$

where $\tilde{u}_{n}(x)$ denotes the approximate solution using $n$-th polynomials while the convergence of nonlinear BVP is calculated by the absolute error of two consecutive iterations such that

$$
\left|\tilde{u}_{n}^{N+1}-\tilde{u}_{n}^{N}\right|<10^{-12}
$$

where $N$ is the Newton's iteration number.

Example 1: Consider the linear differential equation of eleventh-order [7, 8]

$$
\frac{d^{11} u}{d x^{11}}-u=-22(5+x) e^{x}, \quad 0 \leq x \leq 1
$$

subject to boundary conditions:

$$
\begin{gathered}
u(0)=1, u(1)=0, u^{\prime}(0)=1, u^{\prime}(1)=-2 e, u^{\prime \prime}(0)=-1, u^{\prime \prime}(1)=-6 e, u^{\prime \prime \prime}(0)=-5, u^{\prime \prime \prime}(1)=-12 e, u^{(i v)}(0)=-11, \\
u^{(i v)}(1)=-20 e, u^{(v)}(0)=-19
\end{gathered}
$$

The analytic solution of the above problem is $u(x)=\left(1-x^{2}\right) e^{x}$. The maximum absolute errors by the present method are summarized in Table 1. On the other hand, it is observed that the accuracy is found nearly the order $10^{-12}$ in [7] by Siddiqi et al and nearly the order $10^{-13}$ in [8] by Amjad Hussain et al. Now the exact and approximate solutions are depicted in Fig. 1of example 1 for $n=14$.

Table 1: Maximum Absolute Errors for the Example 1

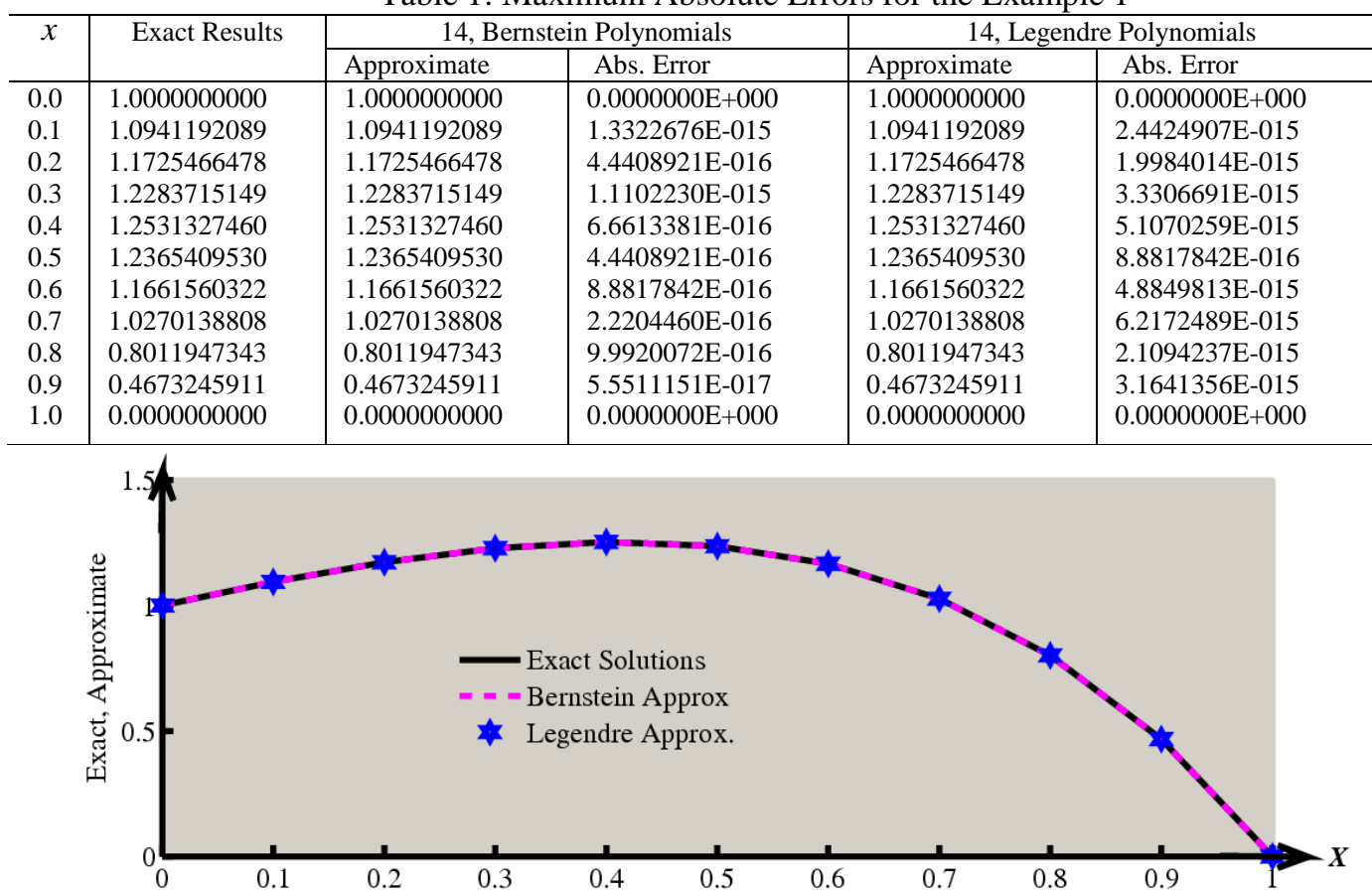

Fig. 1: Graphical representation of exact and approximate solutions of example 1. 
Example 2: Consider the linear BVP [7, 8]

$$
\frac{d^{11} u}{d x^{11}}+u=22(5 \sin x+x \cos x)+\left(1-x^{2}\right)(\sin x+\cos x), \quad 0 \leq x \leq 1
$$

subject to boundary conditions:

$$
\begin{gathered}
u(0)=1, u(1)=0, u^{\prime}(0)=0, u^{\prime}(1)=-2 \cos 1, u^{\prime \prime}(0)=-3, u^{\prime \prime}(1)=4 \sin 1-2 \cos 1, u^{\prime \prime \prime}(0)=0, u^{\prime \prime \prime}(1)=6 \sin 1+6 \cos 1, \\
u^{(i v)}(0)=13, u^{(i v)}(1)=-8 \sin 1+12 \cos 1, u^{(v)}(0)=0 .
\end{gathered}
$$

The analytic solution of the above problem is $u(x)=\left(1-x^{2}\right) \cos x$. The maximum absolute errors, shown in Table 2, are listed to compare with existing results. The maximum absolute errors were found by Siddiqi et al [7] is $5.148 \times 10^{-12}$ and by Amjad Hussain et al [8] is $9.560 \times 10^{-13}$. We have shown the exact and approximate solutions in Fig. 2 of example 2 for $n=14$.

Table 2: Maximum Absolute Errors for the Example 2

\begin{tabular}{c|l|l|l|l|l}
\hline$x$ & Exact Results & \multicolumn{2}{|c|}{14, Bernstein Polynomials } & \multicolumn{2}{c}{14, Legendre Polynomials } \\
\cline { 3 - 6 } & & Approximate & Abs. Error & Approximate & Abs. Error \\
\hline 0.0 & 1.0000000000 & 1.0000000000 & $0.0000000 \mathrm{E}+000$ & 1.0000000000 & $0.0000000 \mathrm{E}+000$ \\
0.1 & 0.9850541236 & 0.9850541236 & $5.5511151 \mathrm{E}-016$ & 0.9850541236 & $5.5511151 \mathrm{E}-016$ \\
0.2 & 0.9408639147 & 0.9408639147 & $4.4408921 \mathrm{E}-016$ & 0.9408639147 & $5.5511151 \mathrm{E}-016$ \\
0.3 & 0.8693562051 & 0.8693562051 & $5.5511151 \mathrm{E}-016$ & 0.8693562051 & $8.8817842 \mathrm{E}-016$ \\
0.4 & 0.7736912350 & 0.7736912350 & $1.1102230 \mathrm{E}-016$ & 0.7736912350 & $1.3322676 \mathrm{E}-015$ \\
0.5 & 0.6581869214 & 0.6581869214 & $4.4408921 \mathrm{E}-016$ & 0.6581869214 & $0.0000000 \mathrm{E}+000$ \\
0.6 & 0.5282147935 & 0.5282147935 & $5.5511151 \mathrm{E}-016$ & 0.5282147935 & $1.3322676 \mathrm{E}-015$ \\
0.7 & 0.3900695155 & 0.3900695155 & $5.5511151 \mathrm{E}-017$ & 0.3900695155 & $1.2767565 \mathrm{E}-015$ \\
0.8 & 0.2508144154 & 0.2508144154 & $2.7755576 \mathrm{E}-016$ & 0.2508144154 & $1.6653345 \mathrm{E}-016$ \\
0.9 & 0.1181058940 & 0.1181058940 & $8.3266727 \mathrm{E}-017$ & 0.1181058940 & $4.7184479 \mathrm{E}-016$ \\
1.0 & 0.0000000000 & 0.0000000000 & $0.0000000 \mathrm{E}+000$ & 0.0000000000 & $0.0000000 \mathrm{E}+000$ \\
\hline
\end{tabular}

Example 3: Consider the following nonlinear differential equation [7]

$$
\frac{d^{11} u}{d x^{11}}=11(\cos x-\sin x)-x(\cos x+\sin x)-u^{2}+x^{2}(1-2 \sin x \cos x), 0 \leq x \leq 1
$$

subject to boundary conditions:

$$
\begin{gathered}
u(0)=0, u(1)=\sin 1-\cos 1, u^{\prime}(0)=-1, u^{\prime}(1)=2 \sin 1, u^{\prime \prime}(0)=2, u^{\prime \prime}(1)=\sin 1+3 \cos 1, u^{\prime \prime \prime}(0)=3, \\
u^{\prime \prime \prime}(1)=-4 \sin 1+2 \cos 1, u^{(i v)}(0)=-4, u^{(i v)}(1)=-3 \sin 1-5 \cos 1, u^{(v)}(0)=-5
\end{gathered}
$$

The exact solution of this BVP is $u(x)=x(\sin x-\cos x)$. Consider the approximate solution of $u(x)$ as

$$
\tilde{u}(x)=\theta_{0}(x)+\sum_{i=1}^{n} \alpha_{i} N_{i, n}(x), n \geq 1
$$

Here $\theta_{0}(x)=1+x(\sin 1-\cos 1-1)$ is specified by the essential boundary conditions in eqn. (18b). Also $N_{i, n}(0)=N_{i, n}(1)=0$ for each $i=1,2, \ldots, n$. Putting eqn. (19) into eqn. (18a), the Galerkin weighted residual equations are

$$
\int_{0}^{1}\left[\frac{d^{11} \tilde{u}}{d x^{11}}+\tilde{u}^{2}-11(\cos x-\sin x)+x(\cos x+\sin x)-x^{2}(1-2 \sin x \cos x)\right] N_{k, n}(x) d x=0
$$

Integrating $1^{\text {st }}$ term of (20) by parts, we obtain

$$
\begin{aligned}
\int_{0}^{1} \frac{d^{11} \tilde{u}}{d x^{11}} N_{k, n}(x)= & -\left[\frac{d N_{k, n}(x)}{d x} \frac{d^{9} \tilde{u}}{d x^{9}}\right]_{0}^{1}+\left[\frac{d^{2} N_{k, n}(x)}{d x^{2}} \frac{d^{8} \tilde{u}}{d x^{8}}\right]_{0}^{1}-\left[\frac{d^{3} N_{k, n}(x)}{d x^{3}} \frac{d^{7} \tilde{u}}{d x^{7}}\right]_{0}^{1}+\left[\frac{d^{4} N_{k, n}(x)}{d x^{4}} \frac{d^{6} \tilde{u}}{d x^{6}}\right]_{0}^{1} \\
- & {\left[\frac{d^{5} N_{k, n}(x)}{d x^{5}} \frac{d^{5} \tilde{u}}{d x^{5}}\right]_{0}^{1}+\left[\frac{d^{6} N_{k, n}(x)}{d x^{6}} \frac{d^{4} \tilde{u}}{d x^{4}}\right]_{0}^{1}-\left[\frac{d^{7} N_{k, n}(x)}{d x^{7}} \frac{d^{3} \tilde{u}}{d x^{3}}\right]_{0}^{1}+\left[\frac{d^{8} N_{k, n}(x)}{d x^{8}} \frac{d^{2} \tilde{u}}{d x^{2}}\right]_{0}^{1} } \\
& -\left[\frac{d^{9} N_{k, n}(x)}{d x^{9}} \frac{d \tilde{u}}{d x}\right]_{0}^{1}+\int_{0}^{1} \frac{d^{10} N_{k, n}(x)}{d x^{10}} \frac{d \tilde{u}}{d x} d x
\end{aligned}
$$

Using eqn. (21) into eqn. (20) and using approximation for $\tilde{u}(x)$ given in eqn. (19) and after applying the conditions given in eqn. (18b) and rearranging the terms for the resulting equations we obtain 


$$
\begin{aligned}
& \sum_{i=1}^{n}\left[\int_{0}^{1}\left[\frac{d^{10} N_{k, n}(x)}{d x^{10}} \frac{d N_{i, n}(x)}{d x}+2 \theta_{0} N_{i, n}(x) N_{k, n}(x)+\sum_{j=1}^{n} \alpha_{j} \int_{0}^{1}\left(N_{i, n}(x) N_{j, n}(x) N_{k, n}(x)\right)\right]\right] d x-\left[\frac{d N_{k, n}(x)}{d x} \frac{d^{9} N_{i, n}(x)}{d x^{9}}\right]_{x=1} \\
& +\left[\frac{d N_{k, n}(x)}{d x} \frac{d^{9} N_{i, n}(x)}{d x^{9}}\right]_{x=0}+\left[\frac{d^{2} N_{k, n}(x)}{d x^{2}} \frac{d^{8} N_{i, n}(x)}{d x^{8}}\right]_{x=1}-\left[\frac{d^{2} N_{k, n}(x)}{d x^{2}} \frac{d^{8} N_{i, n}(x)}{d x^{8}}\right]_{x=0}-\left[\frac{d^{3} N_{k, n}(x)}{d x^{3}} \frac{d^{7} N_{i, n}(x)}{d x^{7}}\right]_{x=1} \\
& \left.+\left[\frac{d^{3} N_{k, n}(x)}{d x^{3}} \frac{d^{7} N_{i, n}(x)}{d x^{7}}\right]_{x=0}+\left[\frac{d^{4} N_{k, n}(x)}{d x^{4}} \frac{d^{6} N_{i, n}(x)}{d x^{6}}\right]_{x=1}-\left[\frac{d^{4} N_{k, n}(x)}{d x^{4}} \frac{d^{6} N_{i, n}(x)}{d x^{6}}\right]_{x=0}-\left[\frac{d^{5} N_{k, n}(x)}{d x^{5}} \frac{d^{5} N_{i, n}(x)}{d x^{5}}\right]_{x=1}\right]_{i} \\
& =\int_{0}^{1}\left[-\frac{d^{10} N_{k, n}(x)}{d x^{10}} \frac{d \theta_{0}}{d x}-\theta_{0}{ }^{2} N_{k, n}(x)+\left\{x(\cos x+\sin x)-11(\cos x-\sin x)-x^{2}(1-2 \sin x \cos x)\right\}\right] N_{k, n}(x) \\
& +\left[\frac{d N_{k, n}(x)}{d x} \frac{d^{9} \theta_{0}}{d x^{9}}\right]_{x=1}-\left[\frac{d N_{k, n}(x)}{d x} \frac{d^{9} \theta_{0}}{d x^{9}}\right]_{x=0}-\left[\frac{d^{2} N_{k, n}(x)}{d x^{2}} \frac{d^{8} \theta_{0}}{d x^{8}}\right]_{x=1}+\left[\frac{d^{2} N_{k, n}(x)}{d x^{2}} \frac{d^{8} \theta_{0}}{d x^{8}}\right]_{x=0} \\
& +\left[\frac{d^{3} N_{k, n}(x)}{d x^{3}} \frac{d^{7} \theta_{0}}{d x^{7}}\right]_{x=1}-\left[\frac{d^{3} N_{k, n}(x)}{d x^{3}} \frac{d^{7} \theta_{0}}{d x^{7}}\right]_{x=0}-\left[\frac{d^{4} N_{k, n}(x)}{d x^{4}} \frac{d^{6} \theta_{0}}{d x^{6}}\right]_{x=1}+\left[\frac{d^{4} N_{k, n}(x)}{d x^{4}} \frac{d^{6} \theta_{0}}{d x^{6}}\right]_{x=0} \\
& +\left[\frac{d^{5} N_{k, n}(x)}{d x^{5}} \frac{d^{5} \theta_{0}}{d x^{5}}\right]_{x=1}-\left[\frac{d^{5} N_{k, n}(x)}{d x^{5}}\right]_{x=0} \times(-5)-\left[\frac{d^{6} N_{k, n}(x)}{d x^{6}}\right]_{x=1} \times(-3 \sin 1-5 \cos 1)+\left[\frac{d^{6} N_{k, n}(x)}{d x^{6}}\right]_{x=0} \times(-4) \\
& +\left[\frac{d^{7} N_{k, n}(x)}{d x^{7}}\right]_{x=1} \times(-4 \sin 1+2 \cos 1)-\left[\frac{d^{7} N_{k, n}(x)}{d x^{7}}\right]_{x=0} \times 3-\left[\frac{d^{8} N_{k, n}(x)}{d x^{8}}\right]_{x=1} \times(\sin 1+3 \cos 1)+\left[\frac{d^{8} N_{k, n}(x)}{d x^{8}}\right]_{x=0} \times 2 \\
& +\left[\frac{d^{9} N_{k, n}(x)}{d x^{9}}\right]_{x=1} \times(2 \sin 1)-\left[\frac{d^{9} N_{k, n}(x)}{d x^{9}}\right]_{x=0} \times(-1)
\end{aligned}
$$

The above equation (22) is equivalent to matrix form

$$
(D+B) A=G
$$

where the elements of $A, B, D, G$ are $a_{i}, b_{i, k}, d_{i, k}$ and $g_{k}$ respectively, given by

$$
\begin{aligned}
d_{i, k}= & \int_{0}^{1}\left[\frac{d^{10} N_{k, n}(x)}{d x^{10}} \frac{d N_{i, n}(x)}{d x}+2 \theta_{0} N_{i, n}(x) N_{k, n}(x)\right] d x-\left[\frac{d N_{k, n}(x)}{d x} \frac{d^{9} N_{i, n}(x)}{d x^{9}}\right]_{x=1}+\left[\frac{d N_{k, n}(x)}{d x} \frac{d^{9} N_{i, n}(x)}{d x^{9}}\right]_{x=0} \\
+ & {\left[\frac{d^{2} N_{k, n}(x)}{d x^{2}} \frac{d^{8} N_{i, n}(x)}{d x^{8}}\right]_{x=1}-\left[\frac{d^{2} N_{k, n}(x)}{d x^{2}} \frac{d^{8} N_{i, n}(x)}{d x^{8}}\right]_{x=0}-\left[\frac{d^{3} N_{k, n}(x)}{d x^{3}} \frac{d^{7} N_{i, n}(x)}{d x^{7}}\right]_{x=1} } \\
+ & {\left[\frac{d^{3} N_{k, n}(x)}{d x^{3}} \frac{d^{7} N_{i, n}(x)}{d x^{7}}\right]_{x=0}+\left[\frac{d^{4} N_{k, n}(x)}{d x^{4}} \frac{d^{6} N_{i, n}(x)}{d x^{6}}\right]_{x=1}-\left[\frac{d^{4} N_{k, n}(x)}{d x^{4}} \frac{d^{6} N_{i, n}(x)}{d x^{6}}\right]_{x=0} } \\
& b_{i, k}=\sum_{j=1}^{n} \alpha_{j} \int_{0}^{1}\left(N_{i, n}(x) N_{j, n}(x) N_{k, n}(x)\right) d x \\
g_{k}= & \int_{0}^{1}\left[-\frac{d^{10} N_{k, n}(x)}{d x^{10}} \frac{d \theta_{0}}{d x}-\theta_{0}{ }^{2} N_{k, n}(x)+\left\{x(\cos x+\sin x)-11(\cos x-\sin x)-x^{2}(1-2 \sin x \cos x)\right\}\right]_{k, n}(x) d x \\
& \left.+\left[\frac{d N_{k, n}(x)}{d x} \frac{d^{9} \theta_{0}}{d x^{9}}\right]_{x=1}\right]_{k=1}-\left[\frac{d N_{k, n}(x)}{d x} \frac{d^{9} \theta_{0}}{d x^{9}}\right]_{x=0}-\left[\frac{d^{2} N_{k, n}(x)}{d x^{2}} \frac{d^{8} \theta_{0}}{d x^{8}}\right]_{x=1}+\left[\frac{d^{2} N_{k, n}(x)}{d x^{2}} \frac{d^{8} \theta_{0}}{d x^{8}}\right]_{x=0} \\
& +\left[\frac{d^{3} N_{k, n}(x)}{d x^{3}} \frac{d^{7} \theta_{0}}{d x^{7}}\right]_{x=1}-\left[\frac{d^{3} N_{k, n}(x)}{d x^{3}} \frac{d^{7} \theta_{0}}{d x^{7}}\right]_{x=0}-\left[\frac{d^{4} N_{k, n}(x)}{d x^{4}} \frac{d^{6} \theta_{0}}{d x^{6}}\right]_{x=1}+\left[\frac{d^{4} N_{k, n}(x)}{d x^{4}} \frac{d^{6} \theta_{0}}{d x^{6}}\right]_{x=0}
\end{aligned}
$$




$$
\begin{aligned}
& +\left[\frac{d^{5} N_{k, n}(x)}{d x^{5}} \frac{d^{5} \theta_{0}}{d x^{5}}\right]_{x=1}-\left[\frac{d^{5} N_{k, n}(x)}{d x^{5}}\right]_{x=0} \times(-5)-\left[\frac{d^{6} N_{k, n}(x)}{d x^{6}}\right]_{x=1} \times(-3 \sin 1-5 \cos 1) \\
& +\left[\frac{d^{6} N_{k, n}(x)}{d x^{6}}\right]_{x=0} \times(-4)+\left[\frac{d^{7} N_{k, n}(x)}{d x^{7}}\right]_{x=1} \times(-4 \sin 1+2 \cos 1)-\left[\frac{d^{7} N_{k, n}(x)}{d x^{7}}\right]_{x=0} \times 3 \\
& -\left[\frac{d^{8} N_{k, n}(x)}{d x^{8}}\right]_{x=1} \times(\sin 1+3 \cos 1)+\left[\frac{d^{8} N_{k, n}(x)}{d x^{8}}\right]_{x=0} \times 2+\left[\frac{d^{9} N_{k, n}(x)}{d x^{9}}\right]_{x=1} \times(2 \sin 1) \\
& -\left[\frac{d^{9} N_{k, n}(x)}{d x^{9}}\right]_{x=0} \times(-1)
\end{aligned}
$$

The initial values of these coefficients $\alpha_{i}$ are obtained by applying Galerkin method to the BVP neglecting the nonlinear term in (18a). That is, to find initial coefficients we solve the system

$$
D A=G
$$

whose matrices are constructed from

$$
\begin{aligned}
d_{i, k}=\int_{0}^{1} \frac{d^{10} N_{k, n}(x)}{d x^{10}} \frac{d N_{i, n}(x)}{d x} d x-\left[\frac{d N_{k, n}(x)}{d x} \frac{d^{9} N_{i, n}(x)}{d x^{9}}\right]_{x=1}+\left[\frac{d N_{k, n}(x)}{d x} \frac{d^{9} N_{i, n}(x)}{d x^{9}}\right]_{x=0}+\left[\frac{d^{2} N_{k, n}(x)}{d x^{2}} \frac{d^{8} N_{i, n}(x)}{d x^{8}}\right]_{x=1} \\
-\left[\frac{d^{2} N_{k, n}(x)}{d x^{2}} \frac{d^{8} N_{i, n}(x)}{d x^{8}}\right]_{x=0}-\left[\frac{d^{3} N_{k, n}(x)}{d x^{3}} \frac{d^{7} N_{i, n}(x)}{d x^{7}}\right]_{x=1}+\left[\frac{d^{3} N_{k, n}(x)}{d x^{3}} \frac{d^{7} N_{i, n}(x)}{d x^{7}}\right]_{x=0} \\
+\left[\frac{d^{4} N_{k, n}(x)}{d x^{4}} \frac{d^{6} N_{i, n}(x)}{d x^{6}}\right]_{x=1}-\left[\frac{d^{4} N_{k, n}(x)}{d x^{4}} \frac{d^{6} N_{i, n}(x)}{d x^{6}}\right]_{x=0}-\left[\frac{d^{5} N_{k, n}(x)}{d x^{5}} \frac{d^{5} N_{i, n}(x)}{d x^{5}}\right]_{x=1} \\
g_{k}=\int_{0}^{1}\left[-\frac{d^{10} N_{k, n}(x)}{d x^{10}} \frac{d \theta_{0}}{d x}+\left\{x(\cos x+\sin x)-11(\cos x-\sin x)-x^{2}(1-2 \sin x \cos x)\right] N_{k, n}(x) d x\right.
\end{aligned}
$$$$
+\left[\frac{d N_{k, n}(x)}{d x} \frac{d^{9} \theta_{0}}{d x^{9}}\right]_{x=1}-\left[\frac{d N_{k, n}(x)}{d x} \frac{d^{9} \theta_{0}}{d x^{9}}\right]_{x=0}-\left[\frac{d^{2} N_{k, n}(x)}{d x^{2}} \frac{d^{8} \theta_{0}}{d x^{8}}\right]_{x=1}
$$$$
+\left[\frac{d^{2} N_{k, n}(x)}{d x^{2}} \frac{d^{8} \theta_{0}}{d x^{8}}\right]_{x=0}+\left[\frac{d^{3} N_{k, n}(x)}{d x^{3}} \frac{d^{7} \theta_{0}}{d x^{7}}\right]_{x=1}-\left[\frac{d^{3} N_{k, n}(x)}{d x^{3}} \frac{d^{7} \theta_{0}}{d x^{7}}\right]_{x=0}
$$$$
-\left[\frac{d^{4} N_{k, n}(x)}{d x^{4}} \frac{d^{6} \theta_{0}}{d x^{6}}\right]_{x=1}+\left[\frac{d^{4} N_{k, n}(x)}{d x^{4}} \frac{d^{6} \theta_{0}}{d x^{6}}\right]_{x=0}+\left[\frac{d^{5} N_{k, n}(x)}{d x^{5}} \frac{d^{5} \theta_{0}}{d x^{5}}\right]_{x=1}
$$$$
-\left[\frac{d^{5} N_{k, n}(x)}{d x^{5}}\right]_{x=0} \times(-5)-\left[\frac{d^{6} N_{k, n}(x)}{d x^{6}}\right]_{x=1} \times(-3 \sin 1-5 \cos 1)+\left[\frac{d^{6} N_{k, n}(x)}{d x^{6}}\right]_{x=0} \times(-4)
$$$$
+\left[\frac{d^{7} N_{k, n}(x)}{d x^{7}}\right]_{x=1} \times(-4 \sin 1+2 \cos 1)-\left[\frac{d^{7} N_{k, n}(x)}{d x^{7}}\right]_{x=0} \times 3-\left[\frac{d^{8} N_{k, n}(x)}{d x^{8}}\right]_{x=1}
$$$$
\times(\sin 1+3 \cos 1)+\left[\frac{d^{8} N_{k, n}(x)}{d x^{8}}\right]_{x=0} \times 2+\left[\frac{d^{9} N_{k, n}(x)}{d x^{9}}\right]_{x=1} \times(2 \sin 1)-\left[\frac{d^{9} N_{k, n}(x)}{d x^{9}}\right]_{x=0} \times(-1)
$$

Once the initial values of $\alpha_{i}$ are obtained from eqn. (24a), they are substituted into eqn. (23a) to obtain new estimates for the values of $\alpha_{i}$. This iteration process continues until the converged values of the unknown parameters are obtained. Substituting the final values of the parameters into eqn. (19), we obtain an approximate solution of the BVP (18). Numerical results for example 3 are shown in the following Table 3. On the contrary the maximum absolute error were found by Siddiqi et al [7] is $4.415 \times 10^{-10}$. We have shown the exact and approximate solutions in Fig. 3 of example 3 for $n=13$. 


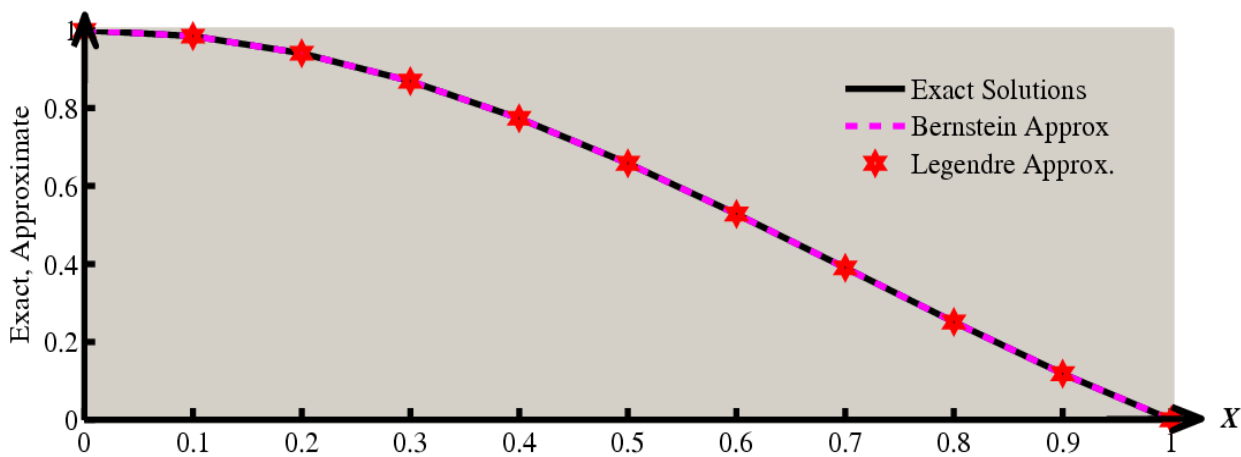

Fig. 2: Graphical representation of exact and approximate solutions of example 2 using 15 polynomials.

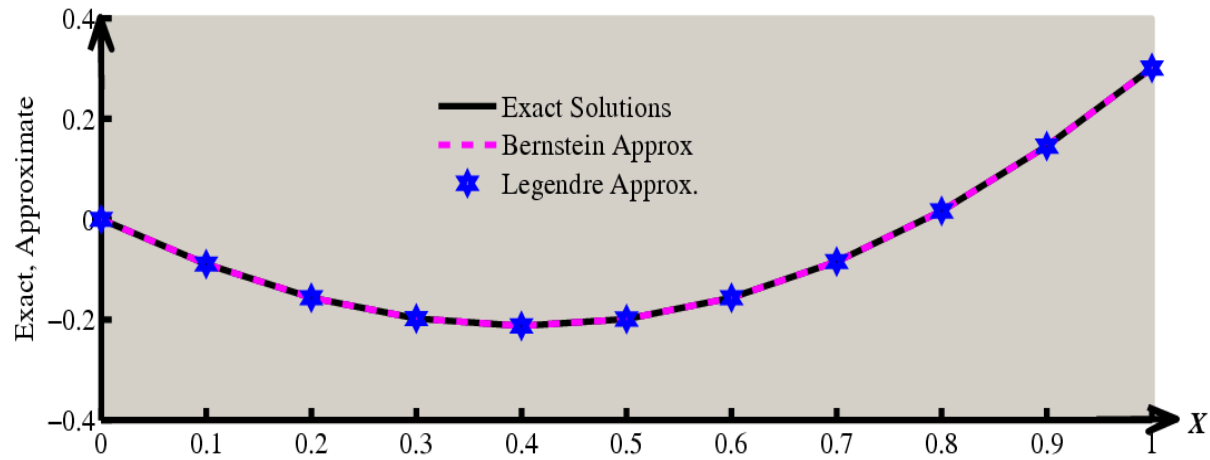

Fig. 3: Graphical representation of exact and approximate solutions of example 3.

Table 3: Numerical Results for Example 3 using 6 iterations

\begin{tabular}{c|l|l|l|l|l}
\hline$x$ & Exact Results & \multicolumn{2}{|c|}{13, Bernstein Polynomials } & \multicolumn{2}{c}{13, Legendre Polynomials } \\
\cline { 3 - 6 } & & Approximate & Abs. Error & Approximate & Abs. Error \\
\hline 0.0 & 0.0000000000 & 0.0000000000 & $0.0000000 \mathrm{E}+000$ & 0.0000000000 & $5.3209018 \mathrm{E}-027$ \\
0.1 & -0.0895170749 & -0.0895170749 & $9.7560848 \mathrm{E}-015$ & -0.0895170749 & $9.7977182 \mathrm{E}-015$ \\
0.2 & -0.1562794494 & -0.1562794494 & $1.0380585 \mathrm{E}-014$ & -0.1562794494 & $1.0602630 \mathrm{E}-014$ \\
0.3 & -0.1979448847 & -0.1979448847 & $2.9445890 \mathrm{E}-013$ & -0.1979448847 & $2.9437563 \mathrm{E}-013$ \\
0.4 & -0.2126570607 & -0.2126570607 & $8.5739749 \mathrm{E}-013$ & -0.2126570607 & $8.5667584 \mathrm{E}-013$ \\
0.5 & -0.1990785116 & -0.1990785116 & $1.3202772 \mathrm{E}-012$ & -0.1990785116 & $1.3194446 \mathrm{E}-012$ \\
0.6 & -0.1564158849 & -0.1564158849 & $1.2643497 \mathrm{E}-012$ & -0.1564158849 & $1.2642110 \mathrm{E}-012$ \\
0.7 & -0.0844371500 & -0.0844371500 & $7.2382378 \mathrm{E}-013$ & -0.0844371500 & $7.2435113 \mathrm{E}-013$ \\
0.8 & 0.0165195052 & 0.0165195052 & $1.9494822 \mathrm{E}-013$ & 0.0165195052 & $1.9536109 \mathrm{E}-013$ \\
0.9 & 0.1455452472 & 0.1455452472 & $2.6478819 \mathrm{E}-014$ & 0.1455452472 & $2.6062486 \mathrm{E}-014$ \\
1.0 & 0.3011686789 & 0.3011686789 & $0.0000000 \mathrm{E}+000$ & 0.3011686789 & $0.0000000 \mathrm{E}+000$ \\
\hline
\end{tabular}

\section{Conclusions}

In this paper, we have used Bernstein and Legendre polynomials as basis functions for the numerical solutions of eleventh-order linear and nonlinear BVPs in the Galerkin method. It is clear from the tables that the numerical results obtained by our method are superior to other existing methods. Also we get better results for Bernstein polynomials than the Legendre polynomials. It may also notice that the numerical solutions are identical with the exact solution even Bernstein and Legendre polynomials are used in the approximation which are shown in Figs. [1-3].

\section{References}

[1]. R.P. Agarwal, Boundary value problems for higher order differential equations (World Scientific, Singapore, 1986).

[2]. J.N. Reddy, Applied functional analysis and variational methods in engineering (Krieger Publishing Co. Malabar, Florida, 1991).

[3]. K.E. Atkinson, An Introduction to Numerical Analysis (John Wiley \& Sons, NY, $2^{\text {nd }}$ Edition, 1989).

[4]. S. Chandrasekhar, Hydrodynamic and Hydromagnetic Stability (Clarendon Press, Oxford, 1961, Reprinted: Dover Books, New York, 1981).

[5]. S. S. Siddiqi and E. H. Twizell - Spline solutions of linear eighth-order boundary value problems, Comput. Methods Appl. Mech. Engrg., 131, 1996, 309-325.

[6]. A. M. Wazwaz - Approximate solutions to boundary value problems of higher order by the modified decomposition method, Comput. Mathe. Appli. 40, 2000, 679-691

[7]. S.S. Siddiqi, G. Akram and I. Zulfiqar, Solution of eleventh-order boundary value problems using variational iteration technique, European Journal of Scientific Research, 30(4), 2009, 505-525.

[8]. Amjad Hussain, S.T. Mohyud-Din and A. Yildirim, A comparison of numerical solutions of eleventh-order boundary value problems, Journal of Information and Computing Science, 7(3), 2012 181-189. 